\title{
Approaches to local SfD provision
}

This chapter provides an in-depth analysis of how SfD is delivered in Zambian communities, paying particular attention to those who are central to this provision; namely, Zambian peer leaders. In doing so, the chapter addresses a number of topics that have frequently featured elsewhere in the SfD literature, in studies that have largely focused on specific programmes. This chapter offers a contrasting and complementary approach that follows Long's (2001) view that the boundaries of specific programmes cannot be clearly demarcated. Therefore, we examine approaches and provision across the range of SfD organizations with which we have undertaken research.

The chapter addresses a number of topics that have frequently featured elsewhere in the SfD literature. In comparison with studies undertaken by others, which have largely focused on specific programmes, it offers a contrasting and complementary approach. Again following Long's (2001) view that the boundaries of specific programmes cannot be clearly demarcated, we instead examine approaches and provision across the range of $\mathrm{SfD}$ organizations with which we have undertaken research. Although most of our work has been conducted with the NGOs EduSport and Sport in Action, it has also involved other SfD organizations operating in Lusaka communities, and further examples are drawn from these. The chapter does not, therefore, attempt to investigate causal relationships between particular SfD programmes and their outcomes, nor does it propose particular models for 'effective' SfD delivery. Instead, by examining SfD practices across different programmes and organizations, it aims to develop fuller knowledge than more narrowly focused research or evaluation can provide.

Throughout the chapter, there is an ongoing focus on the role of peer leaders in SfD delivery. Peer leaders are central to SfD approaches and provision in Zambia, including in the two NGOs with which we have worked most frequently. The involvement of young people in the delivery of SfD is common across the movement, so much so that Nicholls (2009: 167) describes SfD as occurring 'on the backs of peer educators'. However, despite the prominence of 
peer leaders within $\mathrm{SfD}$, there has been little detailed empirical research into their involvement within the academic literature. Specific studies of peer-led $\mathrm{SfD}$ activities have largely been limited to quantitatively evaluating the benefits of this approach for participants (e.g. Maro et al., 2009; Woodcock et al., 2012), leading Coalter (2013: 103) to suggest that the involvement of peer leaders in $\mathrm{SfD}$ may be a 'method in search of a theory'. The intention here is not to examine or develop a particular theory of peer leadership, but to increase knowledge about peer leader attributes, roles and experiences.

The chapter is divided into three main sections. The first considers issues affecting the provision of sports activities in Lusaka communities, the second discusses the delivery of education through and alongside sport, and the third examines associations between SfD provision and community relations. Each section draws on data collected across a number of our studies and makes extensive use of narrative content from adults and young people. Most of the primary data comes from four studies: our initial investigation of the delivery of SfD in Zambia in 2006, when we first collected data from staff and peer leaders at Sport in Action and EduSport; the community-based research led by Iain in 2009; the work undertaken through the Zambian element of the II study, which allowed us to conduct interviews with representatives from a more extensive range of SfD organizations; and the four-year research programme we carried out from 2009, conducted with the Go Sisters programme run by EduSport.

Given the centrality of peer leaders to SfD delivery, the interviews conducted with approximately 50 young people involved in peer leading in the Go Sisters programme are particularly important. Some had been involved as peer leaders for a number of years and several have participated in repeat interviews over this time. During the period of the research we also attended several training sessions for prospective and existing peer leaders, and were present at numerous sport and education activities that they delivered in Lusaka communities. Our informal observations from these activities, and our many informal discussions with EduSport and Sport in Action SfD staff, also inform the chapter.

\section{Provision and peer-led delivery of sport activities}

This section describes the way in which sport is provided by SfD organizations in Zambia and identifies a number of issues that arise in local delivery. Within the literature on $\mathrm{SfD}$ there is surprisingly little detail about the mechanisms of sport provision that underpin SfD programmes, despite warnings such as 
Coakley's (2011) against making uncritical assumptions that sport can contribute to development. The terminology of 'sport for development' could itself be considered indicative of sport as a singular social practice rather than a diverse one. As Spaaij (2009: 1266) emphasizes, however, there are 'important questions about which sports and sports processes produce what outcomes, for which participants and in what circumstances', a point that Coalter (2007a, 2010b, 2013) has consistently asserted. Nevertheless, exploration or even explanation of the nature of sports provision is largely restricted to case studies of specific SfD programmes (e.g. Burnett, 2009; Woodcock et al., 2012), and wider analysis of the particularities of SfD sport provision is limited.

Our examination of sport provision associated with SfD in Zambia shows that Sport in Action, EduSport and other NGOs tend to focus their delivery on a fairly narrow range of sports. Although there are variations in the extent to which particular sports are delivered in specific communities, the majority of provision is based on team sports such as football, netball, basketball and volleyball. Local Zambian indigenous games are also commonly played, either alongside other sporting activities or in stand-alone sessions, typically with younger children in school settings.

Several points are notable about this selection of sports and their link to wider SfD practice and debates. The main sports that SfD NGOs deliver in Zambia are those that have been identified in the SfD literature as raising questions about cultural imperialism (e.g. Hartmann and Kwauk, 2011: 300). While acknowledging this viewpoint, we align more closely with Njelesani et al. (2014) in recognizing the significance of alternative cultural influences, together with a number of important pragmatic considerations. First, the sports delivered by SfD NGOs are those that were already popular and, to differing extents, established in the communities in which Zambian SfD organizations work. The popularity and use of football, in particular, in Zambian communities is reflective of its widespread appeal across the world, and it has been extensively used and valued as an engagement tool in SfD work across the global South (Armstrong, 2004; Spaaij, 2012). Zambian SfD practitioners interviewed by Njelesani et al. (2014: 798) described this sport as being associated with and 'generat[ing] a sense of belonging, collectivity, and identity in Zambian culture'. Although these interviewees recognized the colonial history to which Hartmann and Kwauk (2011) refer, it was this contemporary, localized cultural perspective that had been more important in their decision to use it within their programmes. A second significant local consideration is that, with the exception of basketball, only rudimentary facilities and limited equipment are 
required for the selected sports, which will be seen later to be an especially pertinent issue. Third, team sports enable the engagement of a large number of participants and potentially hold greater potential for integration amongst participants - a rationale that Schulenkorf and Edwards (2012) also identify among SfD practitioners in Sri Lanka.

Another practical consideration in the selection of sports offered by SfD concerns their capacity to enable single- and/or mixed-gender participation. The issues about gender integration/segregation reveal some of the complexities in the interaction between types of sport, local cultural norms and the developmental aims underpinning $\mathrm{SfD}$ activities. Zambian $\mathrm{SfD}$ organizations provide football opportunities, for example, for girls and young women in order to counter specific perceptions of it being a male-only sport, with the intention of also challenging wider cultural stereotypes of gender appropriateness. Such an approach is adopted in EduSport's Go Sisters programme, and has been identified elsewhere by Brady and Banu-Khan (cited in Larkin, 2007) in their study of the MYSA in Kenya. Conversely, some of these same Zambian organizations also deliver provision that conforms to traditional gender divisions - for example, parallel provision of activities in which girls and young women participate in netball alongside boys and young men participating in football. Some of our interviewees have suggested that it can be easier to attract girls and young women into netball; this gender distinction was, however, most visible at inter-school and inter-community competitions delivered by $\mathrm{SfD}$ organizations. This suggests that the norms and practices of other community organizations may also play a part in influencing the form that $\mathrm{SfD}$ provision takes in particular contexts.

Within communities, a range of sport activities are delivered in a variety of settings. The SfD organizations that we engaged with deliver regular activities in a selection of (mainly) community schools, both within the curriculum and as extracurricular sessions. This delivery compensates for the limited capacity of teachers at these schools to deliver PE for all levels of pupils, despite this being made mandatory by a governmental decree in 2006 (Njelesani, 2011). Nevertheless, as Njelesani (2011) also hints, this form of delivery does raise issues regarding piecemeal provision of PE within curriculum time in schools and its substitution by sporting activities. The weekly Friday-afternoon sport tournaments organized by EduSport in Chawama community exemplify these issues. A number of community schools stop all classes so that pupils can take part in the competitive football and netball games that are organized. Some pupils, however, appear to attend only as spectators in what is the designated PE block 
in their schools' timetables. Teachers from the schools that are involved nonetheless value the opportunity for their schools to participate in these tournaments, and a PE teacher in a local government school indicated to us his wish for similar opportunities to be available for his type of school. Such views suggest that the distinction that has been drawn in some global North contexts (e.g. Kirk, 2004), between the inclusive nature of PE and the exclusive nature of competitive sport, may have little relevance to stakeholders in communities in which few other opportunities for organized activity are available.

Other sporting provision is delivered by SfD organizations as communitybased activities. These activities involve regular and semi-regular sport training sessions, and make use of available outdoor pitches and courts in selected communities. Teams developed by EduSport and Sport in Action from their community activities also take part in competitions and leagues, some of which are organized on an inter-community or city-wide basis by these same SfD NGOs. In addition to extending community sport provision by instigating these activities, SfD organizations also initiate or contribute to developing performance pathways for participants to compete at higher levels. During the time of our research, EduSport and Sport in Action were able to offer particularly talented young people further participation opportunities, such as the Norway Cup football tournament, which involve international travel.

The involvement of SfD NGOs in 'mainstream' sport provision in these ways blurs the lines between sport development and sport for development. This is also evident in the work of peer leaders, many of whom see developing sports provision as a key part of their role. Such peer leaders are very proud of how they have increased participation within their communities and have established sporting pathways where young people can now play organized and/or competitive sport. This type of engagement is seen as much more than merely developing sport, and is valued as a way of connecting young people within communities and providing a focus and a sense of pride and identity. These desired outcomes are also integrated within the broader educational aspects of delivery, in which peer leaders facilitate discussions and action with young people about issues that are most pressing to them in their local contexts.

The intertwining of sport development and sport for development is not confined to the work of SfD organizations but is also evident in the aims and approaches of 'sports development' organizations such as community sports clubs. Several representatives of sports clubs in Zambian communities spoke to us about the developmental objectives of their provision; some mentioned only allowing young people to participate in the club's activities if their behaviour 
outside the club met appropriate standards. As yet, the potential contribution that sports clubs in the global South might make to social objectives has not been investigated, in contrast to research conducted in the North (e.g. Coalter, 2007b; Adams, 2011).

Irrespective of the organizations or settings involved, one of the most widespread and common challenges to delivering community sport activities is the limited facilities and equipment available. While Zambian coaches and leaders manage to adapt sports sessions to the resources at their disposal, this is a major obstacle which ultimately limits delivery. A male peer leader from Sport in Action described typical situations:

We used to train with maybe one ball. Sometimes we had two and then you have a session like pass and play, like in volleyball. Then in football we have dribbling and jogging, which is very difficult for the kids to, like, do with one ball. So that was really a challenge.

Similar limitations are common across the global South (Burnett, 2001; Akindes and Kirwan, 2009) and yet are rarely highlighted as priorities, if at all, in global SfD policies (e.g. UN Inter-Agency Task Force on Sport for Development and Peace, 2003; SDPIWG, 2008a). Large-scale international programmes orientated towards facility development within specific countries are also uncommon, although some smaller Northern organizations have supported Zambian SfD NGOs in the construction of new concrete courts and through the provision of equipment.

Although the absence of facilities and equipment can severely impede provision, the real heart of SfD delivery lies in peer leadership. Almost all SfD provision in schools and community settings in Zambia is reliant on the input of local volunteers and, in particular, peer leaders. Despite the centrality of peer leaders within $\mathrm{SfD}$ in the global South generally, there is a lack of SfD research into the characteristics, activities and experiences of the peer leader role. This has, however, been a major focus throughout our own research.

Our findings illustrate that peer leaders in Zambia are far from a homogeneous group. EduSport and Sport in Action had no set age requirement for involvement, and the peer leaders that we interviewed varied from 15 years of age to mid-20s and, in the case of one peer leader, 37 years of age. The gender of these peer leaders broadly reflected the participant base, with a reasonably equal split between young men and young women, which Nicholls (2009) would claim is similar to other SfD organizations and activities in the global South. Most peer leaders live with either their direct or their extended 
family, and thus could be considered to have a greater degree of support than some young people in the communities in which they lived. Peer leaders are therefore diverse in age, experience, home life and ambitions and as a result approach their role from varied perspectives. Acknowledgement of this diversity is often lacking within current literature, in which peer leaders are often discussed as a homogeneous group.

While the responsibilities of peer leading can take up a significant amount of time for the more active peer leaders, all those we spoke to were additionally engaged in other forms of education, employment and volunteering. Some peer leaders had received a scholarship from their NGO to complete their secondary education, but others were often employed in 'piecework', odd jobs with limited financial rewards. Many peer leaders were also volunteering within other organizations, including schools or other NGOs. This wider involvement chimes with a common community-orientated motivation for becoming a peer leader. Molassiotis et al. (2004) found similar motivations amongst peer leaders in another HIV prevention programme in Zambia, and in our study this motivation was exemplified by one female peer leader from Kamwala, who commented:

I gained the love of it and helping people ... looking at my community and wanting to help out and help the people in my community. Even though I am not getting paid, it was wanting to do things for my community.

Such a community-orientated motivation is one of the key factors in recruitment of peer leaders. Other considerations identified by interviewees include a commitment to attending sessions, a willingness to help beyond participating, an ability to foster discussions with young people on broader life issues, and personal 'good' behaviour within the community. The recruitment of new peer leaders is normally undertaken in an informal way, often through identification and recommendation of current participants in sporting activities by existing peer leaders or other community organizers. Some general degree of involvement in sport appeared to be sufficient in terms of sport-specific leadership skills required of new peer leaders.

Once recruited, peer leaders with Sport in Action and EduSport further develop their skills through formal and informal training. Initial training consists of the basics of coaching a range of sports to young people, mainly focusing on rules and organization of team sports as well as some information on traditional games. Subsequently, some peer leaders access more specialized coaching 
training courses that are often based on Northern awards. Interviewees indicated that core elements of these courses are subsequently shared through informal discussion with other peer leaders. It is therefore inappropriate to consider peer leader training solely in the context of formal workshops as most other studies have done (Price and Knibbs, 2009), and instead to understand the upskilling of peer leaders as occurring through a community-of-practice (Wenger, 1999) approach. In this context, young people learn from being members of a peer leader community, through which they have regular contact and discussion with other more experienced individuals.

Furthermore, the formal training that peer leaders engage with could also be considered somewhat variable in content and quality. Whilst peer leaders do engage in formal training and receive ongoing support from the staff of their NGOs, much of the dissemination of knowledge takes place orally and via peer leader networks. Such informal aspects of the peer leadership system can be overlooked in $\mathrm{SfD}$ research undertaken largely by those from the global North. Moreover, the rationalistic desire for standardization and formalization in SfD espoused in some international SfD policies (for example, the International Community Coach Education Standards Framework developed by UK Sport - see Paramasivan, 2013) and research (e.g. Lyras and Welty Peachey, 2011) ignores both the potential rigour and consistency that may exist in informal systems.

The roles that peer leaders have vary considerably. On a day-to-day basis, EduSport and Sport in Action peer leaders are given individual or collective responsibility for the delivery of community sports activities, on a regular or irregular basis. As we shall consider later in the chapter, other peer leaders may take on a variety of tasks, including delivering educative sessions; connecting with local schools, key community stakeholders and parents; and seeking to mobilize young people to come together to try to make significant changes within their communities.

Without the independent contribution of peer leaders, neither Sport in Action nor EduSport would be able to sustain their level of provision across a range of community venues. Moreover, by their own accounts, peer leaders also undertake work to organize competitions, form sports teams and, as the following quote also indicates, initiate particular developments in their communities:

As peer leaders in each zone, we have got our own plans or our own action plans where we plan something, maybe this year, we are going to have this. (Female peer leader, Chawama) 
This level of responsibility certainly contrasts with suggestions in the literature that peer leaders have little control over the organization of the activities they deliver (Price and Knibbs, 2009) and little input into SfD planning (Nicholls, 2009; Njelesani et al., 2014). It is appropriate, therefore, to move away from a global North lens that would place peer leaders as working within formal hierarchical structures headed by SfD NGOs. Certainly in Zambia, the position of peer leaders demonstrates greater complexity and fluidity. Our research points to a gradated peer-leadership model in which those individuals who are no longer at school and have been involved for an extended period of time, some for a number of years, take on further tasks and greater responsibility within the organizations they represent. In the main, such differentiation of roles is absent from the specific literature on peer leaders, although the existence of similar practices elsewhere is hinted at by Coalter, who identifies that:

Many sport-for-development organizations involve young men and women at various levels of planning, implementation and decision making, providing important experience of control, empowerment and a sense of collective responsibility. (Coalter, 2010a: 1381)

The importance of this contribution by peer leaders means that any challenges faced by these individuals are also problematic for SfD organizations and their provision of sporting activities on an ongoing and regular basis. Although the lack of payment to peer leaders by EduSport and Sport in Action does not appear to deter the young people themselves as other studies have suggested (Lesko, 2007), parents and families often do not identify the same value in investing significant time as peer leaders. As the previous chapter showed, young Zambians are commonly expected by their families to undertake family chores, do school work and/or - especially for those who are not at school - be economically productive. These expectations affect peer leaders and the extent to which they can commit their time to regular delivery of activities. One female peer leader from Chawama explained how:

In Africa once your parents send you to school they expect you to help them in return. But once you have finished school, if you do not do anything to help them and get them money then they feel that you are neglecting them or foolish in your mind.

In the first instance, these and other external pressures on peer leaders can affect the regularity with which sports activities are delivered, with resultant implications for the consistent engagement of participants in particular communities. 
Moreover, the high turnover of peer leaders presented further challenges for EduSport and Sport in Action, who had to continually invest time and resources in new peer leaders with little guarantee of their long-term involvement. These issues can take on heightened significance in relation to the development function of the peer leader role, and the next section now considers these aspects.

\section{Delivery of education through and alongside sport}

This section considers how educational activities are delivered through and alongside sport in order to address the complex outcomes to which SfD is orientated in Zambia. The role of peer leaders is, if anything, even more significant to this aspect of $\mathrm{SfD}$, in which relationships between participants and leaders are particularly relevant and important. A peer leadership approach is not only used by SfD NGOs, but frequently by other Zambian NGOs. Svenson et al. (2008) found that $43 \%$ of a sample of Zambian young people aged 15-24 had participated in some form of peer-led education. The common use of peer-led approaches in Zambia and elsewhere is underpinned by a number of both practical and educational considerations. These approaches are frequently justified pedagogically as providing a stimulating way of engaging disadvantaged young people and as enabling a context in which emancipatory education can occur (Warwick and Aggleton, 2004). More practically, peer-led education is relatively cheap to organize, can occur within a community setting and may be more likely to be sustainable than educational activities organized by external agencies (Campbell and MacPhail, 2002).

Peer leaders involved with Sport in Action and EduSport are expected to educate fellow young people on a range of topics. Health-related content tends to dominate, with HIV/AIDS information particularly prevalent. Beyond this, peer leaders usually promote broader health messages, including those about the consequences of drinking, smoking, and other substance or drug misuse. Peer leaders educating young people about HIV/AIDS are expected to provide information on what the virus is, how it is caught and what strategies can be used to avoid infection.

Interventions solely based on the provision of information are unlikely to be effective (Campbell et al., 2005), especially when the ability to resist pressure from peers and partners to have sex is considered to be an essential skill for HIV/AIDS prevention (Hallman, 2005). Zambian SfD peer leaders are therefore also expected to provide a supportive environment that facilitates the development of what are frequently termed 'life skills'. Our interviews across various 
Zambian organizations and individuals involved in SfD indicate rather nebulous ideas about what constitute 'life skills'. As practised by the SfD NGOs and peer leaders in our research, however, life skills can involve supporting young people to develop leadership qualities, improve their negotiation skills and develop strategies for addressing problems in their lives. Peer-led education is, therefore, utilized by Zambian SfD NGOs with the intention of providing information that helps young people identify well-informed choices, and life-skills components that enable them to act on these choices.

Peer leaders in EduSport and Sport in Action deliver SfD activities which are intended to develop both core-content knowledge and broader life skills. They provide core-content information via various different methods. They speak to participants prior to and after sporting activities and also deliver separate education workshops. Also, both sport and traditional Zambian games are adapted to include educational messages as part of their delivery. For example, one of the key educational messages peer leaders are seeking to convey to young people is that it is difficult to tell whether someone is infected with HIV/AIDS by looking at them. It is hoped that this message will encourage young people to take precautions even if they feel a potential partner looks healthy. Zambian peer leaders illustrate this message using a game where a group of participants stand in a line with their hands behind their backs. Several carry a stone in their hands, which symbolizes they are infected with HIV/AIDS. Another participant has to dribble a football along the line and 'guess' who has the stone. Errors in doing so are used as 'teachable moments' to reinforce the key message.

The integration of HIV/AIDS messages into sporting activities is designed to present information in a way that is accessible by young people and appeals to them. Sport-based experiential approaches may be more culturally appropriate than the dissemination of written materials, especially given the high levels of illiteracy and more general lack of engagement with the written word in Zambia (Hughes-d'Aeth, 2002). Adopting a sport-plus approach of disseminating particular educational messages through adapted activities is common across SfD, and many organizations have developed their own curricula to do so. We do not discount Forde's (2014) criticism that the 'neo-liberal' orientation of some such curricula may ignore structural problems in implicitly portraying HIV/AIDS as a problem of individual behaviour. However, the distant lens applied by Forde (2014) in solely analysing curriculum documentation can present an incomplete picture without more localized examination of its practical application. 
For example, consideration must be given to who delivers educational activities within SfD, how they do so, and the training and support they receive. Peer education and leadership are underpinned by a number of pedagogical principles that could make them an effective educational approach. The most frequently cited benefit of peer-led delivery, compared with alternative educational approaches, is that young people may be more responsive to information shared by peers rather than that provided by adults (Turner and Shepherd, 1999; Warwick and Aggleton, 2004). Zambian SfD peer leaders provided several contextually grounded explanations and examples that support this rationale:

I, being a peer leader, will tell them the dangers of having sex at a young age. And they feel comfortable discussing this with me. I had a group [to] who[m] I said: 'How many of you have boyfriends?' And they were all quiet so I said, 'I have, and then they said, 'Oh, we do too.' So it was easier for them to open up and be honest. (Female peer leader, Kamwala)

You can be open to your peers. We are not that open to elders. Like, here in Zambia, in our culture maybe it is a taboo talking with your parents about HIV and AIDS. So it is more easy talking about that to your peers and sharing information about that. (Female peer leader, Kabulonga)

For instance, if a girl is having problems with a boy and he is pressuring her for sex she can discuss that with all of us and we all listen and advise her and support her. That girl knows that she is not alone - she has someone to discuss her problems with. (Female peer Leader, Chawama)

These descriptions reveal a combination of mechanisms by which SfD activities may contribute to HIV prevention. Hughes-d'Aeth (2002) has noted the value of peer leaders being able to share their own experiences with participants and having a 'first-hand' understanding of the community context in which participants live. In Zambian SfD activities, we have observed how peer leaders encourage young people to debate and discuss the critical challenges they face within their lives in order to identify strategies to address these problems. For example, in peer-led Go Sisters sessions, approaches to dealing with peer pressure to have sexual intercourse are commonly addressed. In those sessions observed, peer leaders would explore with participants why this pressure may be inappropriate and what dangers it poses, in order to then encourage the group to suggest solutions for how they may be able to avoid or challenge negative influences from their peers. Participants are encouraged to discuss 'real-life' examples and how they have dealt with these to facilitate learning as experientially as possible.

Peer leaders also spoke of taking their own initiative in organizing and contributing to 'extra' debates beyond the sports context. These debates appear to 
align what Nicholls (2009: 168) identifies as 'the key to successful peer education [that is] horizontal dialogue that enables participants to plan as equals and to take a course of action that is contextually and culturally sound'. One peer leader described facilitating young people to discuss key social issues within their community, identifying possible solutions, and then lobbying parents and community workers to assist them with implementing their ideas. We will discuss the importance and challenges of peer leaders and participants engaging with key stakeholders in the local community in the next section. However, as described by peer leaders, the process of discussion amongst young people appears to encourage them to have a critical awareness of social reality that is necessary for developing agency and transformative action (Freire, 1972), and this is explored further in Chapter 6. There were also indications from peer leaders of resultant action by young people. For example, a group of young participants had identified the high levels of litter as being something that upset them within their community and made their compound feel run-down and dirty. To address this they recruited several others from their sports sessions and held a 'clean-up' in their local area.

Whilst these peer-led approaches to education are theoretically sound and are both pragmatically and intentionally orientated towards the particular community contexts that the SfD NGOs are working in, delivery in Zambia is not without challenges. The aspiration is that peer-led activities provide transformative education experiences that allow young people to be better informed and provide a basis upon which they can make changes within their lives. There is, therefore, a high expectation placed on peer leaders within SfD. Zambian peer leaders recognized that they spend a relatively limited amount of time with participants, whose level of involvement in SfD would vary at different times according to its relative importance in the broader context of their lives. As a result, peer leaders questioned their own ability to engender change within the time spent with participants. With differential involvement of participants, peer leaders also acknowledged that they were unsuccessful at encouraging behaviour change amongst some of the young people they worked with. One female peer leader from Kalingalinga suggested it is a fifty-fifty thing. Others have been changing [for the better] and others they become worse'.

Those peer leaders who sought to develop facilitative approaches discussed how time-consuming and difficult it could be to encourage young people to take action. In addition, at a more fundamental level, it was apparent that some peer leaders struggled to deliver what is a particularly challenging 
educational approach, one that more extensively trained and experienced educators often find difficult. When observing some life skills education taking place, we noticed that peer leaders would often adopt more didactic delivery styles and stand in front of peers giving talks about particular issues with participants remaining passive. Campbell's (2004) study of South African peereducation programmes acknowledged similar issues. This may be a consequence of the lack of guidance included in Zambian SfD peer leader training on facilitation, negotiation and pedagogical underpinnings of participatory education. Various training activities that we have observed over the course of our research have been delivered in a didactic fashion both by senior peer leaders and by staff from SfD NGOs, with limited interaction between the trainers and peers. As such, some young leaders have limited exposure to the types of dialogical approaches acknowledged as more likely to promote transformative thinking and action (Hartmann and Kwauk, 2011). The limitations of training also highlight the accomplishments of those peer leaders who are able to facilitate a more critical approach in their own teaching. Acknowledgement of peer leaders' abilities is rare within the existing literature (Nicholls, 2009) but understanding the training available to them assists with illustrating how exceptional many of the young people who undertake these roles are.

A further challenge identified by peer leaders is the difficulty of establishing a relationship of trust and respect between themselves and the young people participating in the activities. The emergence and selection of peer leaders from groups of participants did ensure a high degree of identification between them, and advocates cite this as a key factor in distinguishing peer-led education from more traditional approaches led by adults, who young people may feel have little understanding of the context of their lives. However, a degree of authority is also required by peer leaders in order to deliver SfD activities and have educational messages accepted by participants. As van der Maas and Otte (2009) also identify in a study of an HIV/AIDS programme in Nigeria, Zambian SfD peer leaders frequently mentioned the challenge of commanding respect from their fellow peers. The following accounts particularly highlight this difficulty when peer leaders are working with young people who they have grown up with and who they are now attempting to exercise some influence over:

At times it is not easy to gain respect; at times you find that some of the people are my age mates, so when you tell them, 'You should do this', they look at you with that eye and sometimes they refuse to do it. (Female peer leader, Kamwala) 
There are some who say, 'I am not listening to you, what do you know?' They think we do not know any more than them. (Female peer leader, Chilenje)

This tension reflects the complexities of social relationships and power dynamics inherent in youth culture in Lusaka (Hansen, 2005). Whilst some of the peer leaders engaged by Zambian SfD NGOs are clearly inspiring role models for many young people, the quotes from the young people above suggest that this may vary depending on the viewpoint of the individual participant. This highlights the importance of carefully matching leaders to particular groups of young people involved in $\mathrm{SfD}$ activities as well as, for future research, the limitations of aggregate measures of the efficacy of peer leadership programmes, as have emerged in the SfD literature (e.g. Maro et al., 2009; Woodcock et al., 2012). Chapter 6 will explore further the perspectives and responses of a variety of young people to the multidimensional peer-led approaches discussed throughout this section.

\section{Community relations and SfD approaches}

This final section considers the extent to which the delivery of SfD in Zambia demonstrates communitarian dimensions. It therefore addresses a particularly prominent criticism within the critical-theoretical strand of literature - the view that SfD policy and practice are underpinned by a neo-liberal orientation that only advances 'individualized notions of success and achievement' (Darnell, 2010a: 66). Hartmann and Kwauk (2011), for example, express concern that SfD practices commonly focus on personal development, to the detriment of the more desirable aim of achieving structural social change. Coakley (2011: 308) voices similar reservations, claiming that advocates of SfD 'discount social issues and the need for progressive change at a collective or community level'.

The issue is complex, however, as failure to directly address community-level change is not the same as ignoring locally important social issues. Enforcing an analytical separation between 'individual' and 'social' change oversimplifies both and ignores their interconnections; certainly authors such as Fokwang (2009), Guest (2009), Spaaij (2012), and Kath and van Buuren (2014), who have spent significant time with local SfD organizations in the global South, depict more complex relationships between $\mathrm{SfD}$, communities and broader social structures. The more internationally focused neo-liberal criticism of the individualism of SfD therefore risks downplaying important aspects of communitarianism that a focus on local practice may reveal. In this section we, therefore, 
consider the extent to which $\mathrm{SfD}$ in Zambian communities is characterized by collective and communitarian dimensions. We again draw on the perspectives of peer leaders, staff from Sport in Action and EduSport, and other community representatives to do so. The discussion falls broadly into two parts - firstly an account of the ways in which SfD organizations demonstrate collectivism and communitarianism within their own work, and secondly an examination of how they engage with external 'communities'.

Focusing on Zambian SfD organizations themselves reveals an immediate communitarian ethos. Our experience of Zambian SfD organizations is that they are premised on collective and communitarian principles. This was emphasized in our very first meetings in 2006 with adults involved with Sport in Action and EduSport, who spoke of their concern to fill the gap for young people in the community who otherwise lacked adult role models and mentors. We have seen above that peer leaders, too, are often motivated to take on their role by a desire to contribute to their own communities. Positive, supportive relationships between young people and adults from these NGOs were seen not only as a positive outcome in themselves but also as facilitating efforts to improve young people's lives in more practical ways:

Our first priority is friendship. Relationships are so much more important even than knowledge. With good relationships so many things can happen. (Staff member, Sport in Action)

The NGOs therefore aimed to contribute to their communities by themselves providing an alternative 'SfD community' for those who might benefit from it. The importance ascribed to this aspiration can be explained through the associated recognition of the breakdown of traditional extended family systems, and community cohesion more generally, as discussed in Chapter 4 .

This communitarian ethos was apparent across the range of sport activities that were identified earlier in the chapter. The potential of sport to 'bring together' young people was viewed as significant by representatives of both SfD NGOs and other community organizations. Rather than being a 'hook' to engage young people in other developmental activities, as in the 'plus-sport' model, there was a common suggestion that the opportunity for young people to meet and interact with one another in community settings was a desired end in itself.

Aspirations for fostering positive relationships and developing supportive communities within SfD are particularly apparent throughout the peer leader system as a whole. As Coalter (2010b) has also identified with the MYSA in 
Kenya, part of the rationale for Zambian SfD NGOs prioritizing peer-led delivery was so that peer leaders could act as positive role models for participants. As a member of staff from Sport in Action put it:

We want our people to be aware [that] sometimes they might be the highest influence on that child's life and they have to act like a parent and a guardian. Give them the love that the children don't have.

There is a reciprocal aspect to role modelling expectations of this type. Peer leaders themselves are affected by their status within communities of participants. As Lesko (2007) also commented upon in her study of a safe-sex campaign in South Africa, SfD peer leaders recognized the responsibilities that came with the role. One Sport in Action staff member who had progressed from being a peer leader spoke of the consequences of his roles in terms that were shared by other interviewed peer leaders:

One thing which keeps me moving, to say I should never disappoint these kids. Because once I screw up in my life or once I do something which won't make a good leader out of them, they'll really get disappointed. And which means others will then quit what they are doing now. ... So if I do something bad, which means it will abolish their lives, it will come to an end. Others will just start doing bad things because of me.

Such responsibilities to their communities of $\mathrm{SfD}$ participants sit differently across a range of peer leaders. In contrast to the individual quoted above, for whom increased responsibility appeared to positively influence his own behavioural decision making, another peer leader referred to problems caused by the lack of positive modelling behaviours amongst some fellow peer leaders. The influence of peer leaders may, therefore, have varied consequences if they are viewed as role models by participants. Turner and Shepherd (1999) reinforce the need for caution in considering the effects of role modelling, which they identify as potentially problematic when linked to attempts to change sexualhealth behaviours.

Peer leaders not only identified responsibilities to their communities of participants but also spoke of the social support that they themselves gain through their involvement as peer leaders. As will be evidenced further in the next chapter, one of the key findings to emerge from the research is the establishment of peer leader networks and 'families'. Peer leaders organized informal meetings between themselves to discuss particular issues in the delivery of sporting and educational activities and develop solutions. This collective support was similar 
to that in another study of Zambian peer leaders (Svenson and Burke, 2005), and was perceived by our interviewees to be invaluable in contributing to peer leaders' own personal development as well as their ability to perform their roles effectively. The description of solidarity amongst a peer leadership 'family' highlights the cultural relevance of this system in a context where the ravages of HIV/AIDS have inflicted significant damage on the traditional, and valued, extended family system. The hierarchical dimensions of the peer leadership system also reinforce the association with traditional family models. A number of peer leaders referred to staff in their respective SfD NGOs as 'parents' and discussed the importance of their ongoing advice and guidance in dealing with particular situations.

As indicated earlier, there are also a number of examples of peer leaders graduating to roles with greater responsibility and, potentially, paid employment within their SfD NGOs. Northern donor funding allowed Sport in Action and EduSport to employ additional staff, who they tended to recruit from peer leaders who had demonstrated significant commitment to planning and delivering activities in their local communities for many years. Even if not formalized and only available to a limited number, the existence of this progression route appeared to be a source of motivation for peer leaders. For example, the same Sport in Action staff member cited earlier recalled his views of the organization's staff when he had been a peer leader: 'we were still looking to up to them, you know this is what I can do. They were my inspiration'.

The role of peer leader brings many benefits to individuals; in fact, as we discuss in the next chapter, peer leaders might be considered some of the main beneficiaries of SfD programmes. Yet as the above accounts illustrate, peer leading is not an individualized activity but one which fosters connections and collective identity and joint working. One important dimension of local SfD organizations, which Coalter (2009) also touches upon, is their creation of these supportive and developmental 'internal' SfD communities in which peer leaders and staff participate. Whether the communitarian ethos of SfD organizations in Zambia is evident elsewhere in Africa and in other regions is not known but is amenable to future research.

This section so far has considered a number of ways in which Zambian SfD organizations demonstrate collectivist values in their own practices and the social relations that they seem to foster within sport. We turn our attention now to a central issue for $\mathrm{SfD}$ - how to work towards social change at a collective and structural level. 
Any efforts to foster social change first require acceptance and support of SfD activities within local communities. Resistance in communities can inhibit the development that staff and peer leaders from SfD organizations seek. In Lusaka, the challenge of engaging young people in SfD activities was exacerbated by sceptical views of sport held by adult community members, as Guest (2009) also recognized in Namibia. Such views were widely commented upon by representatives of SfD organizations, as exemplified by the following quotations:

Most of the parents believe that if their children are just playing football then they will not concentrate at school. (Staff member, EduSport)

One parent has been stopping their child from coming [to sport activities] ... and one parent has been telling [the child] what they are doing is rubbish. (Staff member, Sport in Action)

Peer leaders were also open about the difficulties they had engaging young people and encouraging them to change potentially damaging behaviours when the broader social context was not supportive. These data, therefore, contribute to understanding the influence of broader community and family structures on peer education approaches, an issue that has received limited attention in the literature (Campbell and MacPhail, 2002; Campbell and Mzaidume, 2002; Price and Knibbs, 2009). Peer leaders and staff from SfD organizations identified how these contextual influences could severely impinge on the ability of peer-led education to promote changes in attitudes, values and behaviours amongst young people, even when they were committed to adopting health-protecting behaviours. As this male peer leader from Kabulonga explained:

If the family is not in agreement with our views and the youth is determined that he will behave as he is doing, then we may not change that. We have to keep on and try but without the family it is not easy.

Another peer leader explained how the impact of SfD activities would be limited in cases of young women and girls being forced into prostitution by their families:

Some girls, they just have no choice; they may not want to behave immorally, but their father or their brothers are forcing them into prostitution so they have money. They tell the girl, 'You have to get us money,' and if she says no she will not do it they beat her up and make her do it. For that girl, learning with us does not really help, it does not change. (Female participant, central Lusaka) 
Issues of trust within communities compounded the challenges that $\mathrm{SfD}$ and other organizations faced. Historically and more broadly, NGOs and CBOs have been associated with problems of corruption (Bawa, 2013) and short-termism (Samoff, 2004). In turn, this has affected the credibility of other organizations within communities, as Fokwang (2009) also identified in her study of Ntambag Brothers Association in Cameroon. Many of our community-based interviewees spoke of similar difficulties. For example, as a representative of an SfD NGO in Chawama described:

[there were] problems before we had a chance to speak to these parents. ... Because when you go out, some of the parents will say: 'Ah, they are making money. These guys are gaining money and then they are using our children.'

As a result, SfD organizations undertook specific activities aimed at promoting engagement between themselves, peer leaders and family members. For example, as part of the Go Sisters programme peer leaders organize regular parents' workshops, which seek to encourage members of the broader community to value SfD activities and to support the desired outcomes of peer-led activities. Peer leaders and community representatives provided examples of how some parents were modifying their attitudes:

At first, when it started, they used to have that; they say, 'Sports, maybe they're just for men, but this time they've come to understand the important of sports for their children, yeah. This time there's no problem. (Female peer leader, Chawama)

Most of the girls that have really taken part in Go Sisters, they do tell their parents about what is happening in Go Sisters. And most of the parents are really proud of their girls, you know, being part of the Go Sisters, and then telling them whatever is really happening in Go Sisters. So it is really, you know, making an impact, even to the family members, even the other girls that are around us. (Female peer leader, Chawama)

Many people say that it's a very good programme and they should continue funding it, do more sports facilities, yes. It's, in Zambian tradition, people believe that girls are supposed to be in the kitchen only, but they now realize they are supposed to do something else - like gender, like gender equality. (Female peer leader, Chawama)

Most of them, the parents, they are admiring what is happening ... most of them, they've appreciated because looking at the way their kids used to be in the past, and the lives that they are leading this time is different, yeah. (Teacher, community school, Chawama) 
However, NGO staff also suggested that parents who engaged with workshops and the programme more generally tended to be those already inclined to support their children and that efforts by peer leaders were not necessarily reaching the parents who posed the greatest obstacle to positive change.

SfD peer leaders felt relatively powerless to do anything if parents remained unsupportive. Broader community and family structures are, therefore, critical to facilitating positive impact, but how these interact with peer-led education has rarely been considered in the existing literature (Campbell and MacPhail, 2002; Campbell and Mzaidume, 2002; Price and Knibbs, 2009). Power dynamics between adults and young people that were illustrated in the previous chapter made negotiating with parents particularly difficult for some young peer leaders, as one explained:

When a parent says that a girl cannot come, I try to speak with them and explain what we do. They will not always listen to me though; to them I am just a child and I have no right to be saying these things to them. (Female peer leader, Chawama)

Therefore, peer leaders' attempts at engagement with adults within their communities came with the risk of clashing with traditional cultural values and beliefs. Young people are generally expected to show unquestioning respect for adults. A fine balance can therefore be identified between empowering both peer leaders and young participants and becoming 'over-empowered', as was captured in the following explanation by a female peer leader from Kabwata:

So, sometimes it makes them disrespectful to their parents, because they think they have the right to do anything and that is not true. If they get into fights with parents, they say, 'You can't tell me that, I know my right,' and then the parents get angry, saying, 'This is what they are teaching you, to disrespect your elders?' You have to balance what you are teaching them.

In a similar fashion, Campbell and MacPhail (2002) also discuss how South African peer leader programmes were heavily criticized by adult community members for encouraging young people to challenge their knowledge and be disrespectful of their authority. Although largely unexplored in the SfD literature, there is a significant challenge for SfD organizations in navigating potential tensions between increasing community support and encouraging the development of young people in ways which could provoke significant challenge to broader cultural traditions, such as respect for elders. 


\section{Conclusions}

This chapter has examined approaches to provision of $\mathrm{SfD}$ in communities in Lusaka. In contrast to empirical case studies that tend to focus on specific programmes, we have attempted a more holistic examination of the delivery of SfD in Zambian communities, and have made extensive use of the knowledge of local adults and young people to inform this. Overall, we have identified great diversity, complexity and fluidity in SfD practices, strongly oriented to the conditions and needs of their immediate local communities. While we do not claim generalizability for these findings, they do offer some additional insights to current understandings of SfD in the academic and policy literature.

The chapter has emphasized how strongly the practical delivery of SfD by NGOs is shaped by local factors, and has especially highlighted the significance of the personal individual influence of peer leaders. In Zambia, as more widely in $\mathrm{SfD}$, peer leaders are the main facilitators of $\mathrm{SfD}$ provision and their varied characteristics, biographies and capacities shape its delivery in multiple ways. As a result, provision at community level combines responsiveness to local needs with pragmatism, such as in the development of provision in locations where peer leaders live and can capitalize on their local knowledge and networks. Peer leaders negotiate constrained conditions and draw on shared local knowledge and expertise through the communities of practice they develop. All of these factors reinforce the significance of local conditions and contexts in shaping SfD.

The resulting forms of provision defy easy categorization, frequently including considerable development of sport itself while also blurring distinctions between 'sport plus' and 'plus sport'. From a local perspective, however, it is precisely this malleability of sport which makes it a valued developmental tool: the choice of sports activities can be shaped according to the availability of facilities and resources, and the development content oriented to the most relevant local issues. As their own accounts show, however, peer leaders' ability to influence individual young people and promote wider social change can face considerable difficulties in the context of broader social and environmental factors.

Despite the strength of the communities established through peer-led networks, these continue to have limited power to encourage broader structural changes of the type needed to alter the material conditions of young people's lives. Whilst the networks are important to young people and assist them with 
navigating the challenges of their everyday lives, they are not necessarily or always sufficient to provide a basis for collective action which might promote structural changes in the local community. Our data especially highlights the critical ways that broader social and environmental contexts affect how much influence individual peer leaders can exert on young people in their communities. Yet our data showed also that changes in wider social networks might indeed be occurring, with previously restrictive views being modified as families became receptive to the involvement of their children in SfD programmes. Such dimensions should not be overlooked and will benefit from fuller research.

In summary, our examination of deliverers and delivery of SfD in Zambia offers a counterpoint to a number of perspectives in the existing literature. First, it is important to identify the contrast between the rationalistic approaches to SfD suggested in the literature (e.g. Lyras and Welty Peachey, 2011; Schulenkorf, 2012) and the complexity and fluidity that characterize peer-led delivery. In the practice of SfD in Zambia, there is no standardized 'formula', and how delivery takes place is influenced by factors associated with SfD organizations, individual peer leaders and the communities in which they operate. Second, the literature has commonly identified and criticized an individualistic focus of SfD practice which, it is suggested, does not address broader community change (Darnell, 2010a; Coakley, 2011; Cornelissen, 2011). While issues of impact are addressed in the following chapter, we can draw similar conclusions to those of Svenson and Burke (2005: 45), who identify that peer-led initiatives can be both 'a product and a method of community mobilization'. SfD approaches and provision in Zambia clearly reflect, are influenced by and, in some respects, challenge local community beliefs, needs and issues. A third broad point of contrast is with the widespread - if not always empirically supported - recognition in the literature of Northern dominance over the practice of SfD. While not denying Northern influence, our research does clearly point to the ways in which local SfD is strongly orientated towards and shaped by the communities in which it is delivered.

In addition to offering these substantive findings, the chapter also provides a further example of the use of local narratives. As in Chapter 4, we have found that data obtained from adults and young people reveal detail and complexity about local realities that would otherwise have remained unknown. The accounts given in this chapter were, however, especially notable for the extent to which they acknowledged and openly discussed negative aspects of SfD, including the constraints within which activities were provided, and the challenges peer 
leaders faced in their roles. As we have shown, young people were willing to discuss with us the difficulties they faced in attempting to generate 'change', whether in individual behaviour or in wider cultural norms, and to describe in some detail their own limited status and authority. Such insightful and selfcritical accounts offer reassurance to those doubtful of the value and integrity of local SfD narratives. The next chapter now makes further use of such data to explore the experiences of SfD from participants' perspectives. 